\title{
Hemispheric low-grade gliomas
}

\author{
Gianpiero Tamburrini ${ }^{1} \cdot$ Jose Hinojosa $^{2}$
}

Received: 8 July 2016 / Accepted: 11 July 2016

(C) Springer-Verlag Berlin Heidelberg 2016

The common concept of low-grade gliomas as benign tumors that in principle might be cured has found its limit in real life in the frequent involvement of eloquent areas of the brain, the frequent intraoperative evidence of tissue with macroscopic aspects not easily distinguished from the surrounding normal brain, and the difficulties of eventually needed complimentary treatments because of the relative resistance of a not negligible part of lowgrade gliomas to both chemotherapy regimens and radiotherapy.

In the last decade, efforts have been therefore directed in three directions. The first is towards improving our preoperative and intraoperative planning through more advanced MR imaging (DTI sequences and functional MR studies), a more extensive use of intraoperative mapping and monitoring in order to reduce the risk of postoperative neurological sequelae, and the attempts to apply the techniques of awake surgery also to the pediatric population.

The second significant effort has been made in the field of intraoperative imaging. Neuronavigation based on preoperative imaging has indeed demonstrated its limits for the intradural steps of the surgery for this kind of lesions, especially when located far from the midline. Conventional- and contrast-enhanced ultrasounds have been widely utilized, presenting the advantage to be the tools of contained cost and easy practical application, but the limit of being operator dependent. Intraoperative MR is nowadays increasingly utilized

Gianpiero Tamburrini

gianpiero.tamburrini@unicatt.it

Jose Hinojosa

jose.hinojosa@salud.madrid.org

1 Pediatric Neurosurgery, Institute of Neurosurgery, Catholic University Medical School, Largo “A. Gemelli”, 8, 00168 Rome, Italy

2 Servicio de Neurocirugía, Hospital Universitario 12 de Octubre, 28041 Madrid, Spain for extending safe removal for low-grade gliomas in the pediatric population as it does not bear the risk of intraoperative radiations, compared with intraoperative CT scan, and its usefulness and reliability. However, the high cost constitutes a significant limit, especially for pediatric neurosurgery centers.

The third direction to improve the management of children with low-grade gliomas certainly involves a better understanding of both the genetic and molecular nature of these tumors, in the hope to achieve results similar to that obtained for malignant tumors (e.g., medulloblastomas). Indeed, starting from the knowledge of genetic syndromes associated with lowgrade gliomas, related molecular pathways are increasingly demonstrating their possible involvement as targets for ultraselective individualized treatments.

Thanks to the abovementioned efforts and advances, more extensive removals of low-grade gliomas have become nowadays possible not only for tumors developing in eloquent cortical areas but also for deep-sited lesions, e.g., insular and thalamic tumors, to which more conservative approaches were offered for a long time, to preserve the quality of life.

Actually, although it is well known that even larger tumor residuals might remain stable for a long time after surgical treatment, the prolonged postoperative observation demonstrates that the extent of tumor resection (EOR), even for low-grade gliomas, is a significantly positive survival factor related to both longer overall survival (OS) and progressionfree survival (PFS). Moreover, it is well stated that the EOR is significantly related to the possibility of epilepsy control in children presenting with seizures.

The annual issue of this year is an attempt to give an overview of the achieved knowledge in the abovementioned three directions, keeping also an eye to possible fields of further knowledge on these tumors which can lead to their further better management, such as the possibility to predict the tumor grade based on preoperative selective neurocognitive evaluations up to possible alternatives (e.g., MRI-guided laser tumor ablation ) to open surgery and advanced complimentary treatments. 